\title{
CNS Anticancer Drug Discovery and Development: 2016 conference insights
}

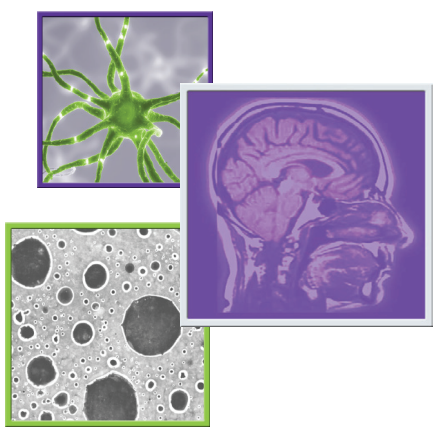

\author{
Victor A Levin ${ }^{* 1,2}$, Lauren E Abrey33, Timothy P Heffron ${ }^{4}$, Peter J Tonge ${ }^{5}$, \\ Arvin C Dar ${ }^{6}$, William A Weiss ${ }^{7} \&$ James M Gallo ${ }^{8}$ \\ CNS Anticancer Drug Discovery and Development \\ 16-17 November 2016, Scottsdale, AZ, USA
}

The 2016 second CNS Anticancer Drug Discovery and Development Conference addressed diverse viewpoints about why new drug discovery/development focused on CNS cancers has been sorely lacking. Despite more than 70,000 individuals in the USA being diagnosed with a primary brain malignancy and 151,669-286,486 suffering from metastatic CNS cancer, in 1999, temozolomide was the last drug approved by the US FDA as an anticancer agent for high-grade gliomas. Among the topics discussed were economic factors and pharmaceutical risk assessments, regulatory constraints and perceptions and the need for improved imaging surrogates of drug activity. Included were modeling tumor growth and drug effects in a medical environment in which direct tumor sampling for biological effects can be problematic, potential new drugs under investigation and targets for drug discovery and development. The long trajectory and diverse impediments to novel drug discovery, and expectation that more than one drug will be needed to adequately inhibit critical intracellular tumor pathways were viewed as major disincentives for most pharmaceutical/ biotechnology companies. While there were a few unanimities, one consensus is the need for continued and focused discussion among academic and industry scientists and clinicians to address tumor targets, new drug chemistry, and more time- and cost-efficient clinical trials based on surrogate end points.

First draft submitted: 15 March 2017; Accepted for publication: 23 March 2017; Published online: 18 July 2017

The second CNS Anticancer Drug Discovery and Development Conference (CADDDC) was held on 16-17 November 2016, before the Society for Neuro-Oncology Annual Meeting. Motivation for CADDDC in 2014 and 2016 was the unfortunate reality that no new drugs had been discovered/developed for primary or secondary CNS tumors. In 1999, temozolomide (TMZ) was the last drug approved by the US FDA as an anticancer agent for high-grade gliomas, although bevacizumab received FDA regulatory approval for the treatment of recurrent/progressive glioblastoma (GBM) in 2009. Following the 2014 CADDDC, a comprehensive white paper was published to provide guidance to the field and, hopefully, to stimulate greater interaction

\section{KEYWORDS}

- gliomas

- medulloblastoma

- chemotherapy • academia

- pharmaceutical industry

- neuro-oncology

'Department of Neurosurgery, University of California San Francisco, San Francisco, CA, 94143, USA

2Department of Neuro-Oncology, The University of Texas MD Anderson Cancer Center, Houston, TX 77030, USA

${ }^{3}$ Hoffman-La Roche Ltd, Basel, Switzerland

${ }^{4}$ Senior Scientist, Genentech, South San Francisco, CA 94080, USA

${ }^{5}$ Department of Chemistry, Stony Brook University, Stony Brook, NY 11794, USA

${ }^{6}$ Oncological \& Pharmacological Sciences, Mount Sinai Icahn School of Medicine, New York, NY 10029, USA

${ }^{7}$ Department of Neurology, University of California San Francisco, San Francisco, CA 949143, USA (W.A.W.), CA, USA

${ }^{8}$ Albany College of Pharmacy \& Health Sciences, Albany, NY 12208, USA (J.M.G.)

*Author for correspondence: Tel..+1 415464 9326; vlevin11@gmail.com

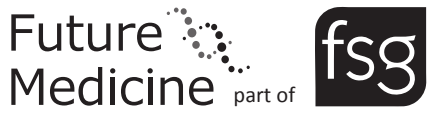


between academia and the pharmaceutical/ biotechnology industries [1-3].

Among the new topics addressed in the second CADDDC were economic factors and pharmaceutical risk assessments, regulatory constraints and perceptions, and need for better imaging surrogates of drug activity. Also included were modeling tumor growth and drug effects in a medical environment where direct tumor sampling for biological effects can be problematic, potential new drugs under investigation, and novel targets for drug discovery and development.

For this overview article, we asked the moderators of each session to summarize their presentations and the 30-45-min discussions that followed each session.

\section{Clinical reality \& pharmaceutical challenges}

We continue to have many unmet clinical challenges and treatment options for patients with CNS cancers. Approximately 70,800 people in the USA are diagnosed with a primary malignant brain tumor each year [4], representing $1.4 \%$ of all cancer diagnoses and $2.4 \%$ of all cancer deaths. Metastatic disease in the CNS is severalfold more common, with incidence estimates of 9-17\% of cancer patients or 151,669-286, 486 patients annually [5].

In the last decades, the critical signaling pathways of GBM have been described, demonstrating RTK/RAS/PI3K pathway alterations in $88 \%$ of patients, $\mathrm{p} 53$ alterations in $87 \%$ and $R B$ alterations in $78 \%$ of patients [6]. Molecular markers, including IDH mutation, chromosome $1 \mathrm{p}$ and 19q status, ATRX mutation and TP53 mutation have sharpened diagnostic precision and treatment paradigms for adult glioma patients [6]. Similar advances have elucidated the underpinnings of medulloblastoma, the most common pediatric tumor, which can now be classified into at least four clinically relevant subtypes based on molecular and genetic features [7].

Unfortunately, these scientific advances have not been translated into development of effective new drugs. This contrasts with progress in the broader field of oncology where multiple targeted agents and immunotherapy have revolutionized the treatment and clinical trajectory for patients with various systemic malignancies. Pharmaceutical challenges and unique considerations pertaining to adequate CNS delivery and exposure are likely to play a critical role in the failure of these systemic agents to change the outcomes for most primary and metastatic brain tumor patients. Methotrexate was one example in the conference. Methotrexate is an early generation drug used in CNS leukemia and primary CNS lymphoma. It been studied using unbound brain extracellular fluid, cerebrospinal fluid and plasma drug levels under dynamic conditions to create informative mathematical models and system-based pharmacokinetic models to define the relationship between brain extracellular fluid and cerebrospinal fluid concentrations [8].

It is not sufficient for a drug simply to reach its cellular target; it must arrive at its target of interest in the CNS tumor with sufficient concentration and residence time to exert its intended effect on tumor cells. Key considerations include: dosing, plasma concentration over time, blood-brain barrier (BBB) transport and target tissue free drug concentration over time, nonspecific binding of drug, target binding kinetics, cellular signal transduction, homeostatic feedback and influence of the disease on the microenvironment. It has become increasingly feasible to represent these factors using a combination of animal and mathematical models to predict effective exposure $[8,9]$.

Beyond having the drug reach the brain tumor target, the kinetics of the specific interaction between the drug and its target is critical. Drug target residence time is an important predictor of efficacy, but often it is not adequately considered by clinicians or pharmaceutical companies. Maximizing target engagement and minimizing off-target binding determines a drug's potency, selectivity and safety. Drugs vary in drug-target residence time, which can be measured by various ways or may be irreversible (e.g., ibrutinib that targets Bruton's tyrosine kinase and afatinib that targets EGFR and erB-2 receptor tyrosine kinases). A long residence time correlates with increased target engagement at low drug concentration, in principal enabling drugs to be dosed less frequently, thereby improving safety. Pharmacokinetic (PK)/pharmacodynamic (PD) models that incorporate drug-target kinetics can best predict clinical effect as most standard approaches assume rapid equilibrium between drug and target despite the nonequilibrated environment in the human body $[10,11]$. This approach sheds light on target vulnerability, the proportion of the target that needs to be inhibited to impact cell growth or survival. Low target vulnerability means that most/all target molecules must be 
inhibited, whereas high vulnerability implies that the desired effect can be obtained by only engaging a fraction of the target.

Protein kinase inhibitors comprise the largest group of targeted drugs used to treat cancer. More than 32 have been approved for systemic malignancies $[12,33]$. Unfortunately, not one has shown consistent efficacy against CNS tumors despite several achieving relatively high brain concentration. One reason contributing to this failure is that the free drug concentration (as opposed to the total drug concentration commonly reported) at the site of action is the species that exerts pharmacological activity. Furthermore, active efflux transport P-gp and BCRP transporter are primary limitations in attaining free CNS exposure for small molecule drugs. Our assessment is that most kinase inhibitors available for clinical use do not have the properties required to achieve adequate free concentrations in the brain. However, while an added barrier to the already significant challenge of discovering a suitable drug candidate, physicochemical properties can be optimized by the medicinal chemist during the drug-discovery phase to develop or select brain penetrant kinase inhibitors. Furthermore, elucidating individual successes such as alectinib (targets ALK) [13] and brigatinib (targets ALK and EGFR receptor kinase) [14] for the treatment of non-smallcell lung cancer brain metastases may encourage development of better therapies for metastatic brain tumors. It is encouraging that some pharmaceutical companies have created a small number of kinase inhibitors for cancer treatment while specifically considering the ability of the new small molecule drugs to freely penetrate the BBB.

In the clinic, sensitive PD biomarkers and imaging techniques have become useful for assessing the subclinical effects of drugs and surrogates to accelerate clinical trials. One example, PET, is applied to assess glucose consumption and glycolytic flux that are regulated by oncogenic signaling and that can be inhibited by some drugs. Thus, it might be possible to identify tumors and/or patients in which a specific tyrosine kinase inhibitor creates an early metabolic change indicative of activity and potential susceptibility to sequential or combination therapies. Another example may be the ability to calculate rates of change in tumor volume using MRI, a technique under study in clinical trials of IDH inhibitors. Traditional end points such as progression-free survival and overall survival (PFS and OS) might not be appropriate for some rare tumors because of the cost and time requirements to complete clinical trials. In addition, new quantitative imaging measures may help guard against discarding active agents that can be used in drug combinations.

\section{Economic \& regulatory challenges}

Many believe that drug development aimed at primary CNS cancers in adults and cancers is a low priority. Large and small pharmaceutical (and biotech) companies have mostly ignored CNS cancer drug discovery/development to limit the financial risk of failure [15]. One argument for why this has occurred is that current USA patent law may not provide sufficient incentives for long-term high-risk cancer research and drug development (R\&D). R\&D investments are greatest for recurrent rapidly growing cancers and decreased for metastatic tumors, lower for regional and localized tumors, and even lower for prevention of cancer. The current FDA approach focuses on survival, resulting in greater expense than surrogate end-point studies since it takes longer to achieve the end point.

Historically, drug development in hematologyoncology has been easier and less costly because biological measures are easy to obtain and can assist in clinical trial design and outcomes measures. This has led to marked improvements in 5-year survival rates during the 30-year period from 1973 to 2003. In comparison, calculations for nonhematological cancers (e.g., gliomas and medulloblastoma) using 2003 data showed that approximately 890,000 life-years were lost at a cost of US $\$ 89$ billion due to a lack of therapies for these patients [15].

The public does not benefit from new drugs until an entity (almost always a pharmaceutical company) demonstrates their safety and efficacy in clinical trials. In general, pharmaceutical companies will not finance trials without lengthy monopoly protection - that is, protected by law from competition. This caveat is primarily because $\mathrm{R} \& \mathrm{D}$ and clinical trials are expensive, risky and time consuming. In addition, pharma views generic competitors as purloining a free ride on the backs of their investments. Therefore, the public would be wise to offer adequate monopoly protection for any new (i.e., not yet FDA approved) drug once a company demonstrates drug safety and efficacy in clinical trials and the FDA approves it.

Regulatory exclusivity periods are: 3 years for previously approved active ingredients, 5 years 
for new active ingredients, 7 years for orphan indications and 12 years for biologics (genetically engineered proteins derived from human genes). These parameters may be too short for small molecule drugs, since pharmaceutical companies screen drugs that cannot be patented out of their pipeline. One solution to the risk/ benefit dilemma for rare and slow growing CNS cancers might be to encourage legislative action to lengthen existing exclusivity periods to the optimal monopoly length for new drugs given in the 2013 MODDERN Cures Act (H.R. 3116) and the 2014 Dormant Therapies Act (S. 3004), which grant 15 years' exclusivity, but only if drugs treat an unmet medical need. All patent rights to the drug are waived after the 15 years of exclusivity ends (i.e., no 'evergreening'). Evergreening refers to producers who extend their patents that are about to expire by using various legal, business and technological strategies.

Primary CNS cancers are considered to be rare diseases on the basis of the definition that this tumor type affects less than 200,000 patients in the USA [16]. In the USA, Orphan Drug Designation provides 7-year exclusivity, tax credits, waiver of application fees and some FDA assistance. In Europe, the European Medicines Agency provides a more generous, 10-year, exclusivity for drugs that are effective against rare cancers. However, the FDA offers competitive grant support for the development of products to diagnose or treat rare diseases through the Office of Orphan Products Development. In this regulatory environment, the challenges for investigators seeking drug development for rare diseases are: small and heterogeneous populations, incompletely characterized natural history of the disease, unfeasible end point(s), limited patient and financial resources, and potential need for global involvement.

For rare cancers, like gliomas and medulloblastoma, how to power small patient sample sizes is problematic while designing clinical trials because the FDA requires the same standards to be included in clinical trial end points that are used for nonorphan disease drugs. For most cases of glioma and medulloblastoma, these standards require some measures of drug activity such as objective response rate, PFS and/or OS benefit. For these rare tumors, demonstrating drug efficacy in a clinical trial can lead to one or more of the following: fast-track designation, breakthrough therapy designation, priority review and accelerated approval to help move drug approval forward quickly when appropriate.
Other views on the challenges for developing novel therapies for CNS cancers from the perspective of pharmaceutical companies were articulated at the conference with some optimism since 17 drugs received regulatory (FDA) approval for oncology in 2015, and seven drugs were approved in 2016. On the other hand, multiple drugs were approved for renal cell cancer, multiple myeloma and non-small-cell lung cancer and most of these approvals were led by large companies. In addition, many of the approvals were for antibody drugs rather than small molecule drugs and none were approved for CNS cancers.

Factors considered important in drug development and that impact how large pharmaceutical companies view their commitment to new cancer therapies are: financial factors, risk aversion and scientific excellence. Financial factors relate to the cost of development and how big the target market size for the drug might be. Risk aversion is based on regulatory constraints, clinical benefit and clinical risk (side effects and unintended consequences of the therapy). The third element is scientific knowledge; viewpoints diverge on how this is considered. For example, large pharmaceutical companies consider whether the therapy will be first-in-class, best-in-class, if it supports the strategy of the company, and if the therapy differentiates the company from its competitors. While these are formidable concerns, some from pharma feel that financial disincentives are not major considerations despite the high cost of drug development. This is, however, a viewpoint that contradicts general perceptions. The market for GBM is approximately 28,000 in the USA and Europe, with potential peak sales of between US\$1.5-3 billion/year depending on treatment efficacy. The forecast for much less common mid- and lower-grade gliomas and medulloblastoma is not nearly as encouraging from a company perspective as the market for medulloblastoma might be less than 1000 .

In addition, many in the pharmaceutical industry consider drugs used to treat malignant brain tumors too risky to pursue. CNS toxicity is a disincentive to develop small molecules capable of $\mathrm{BBB}$ penetration. Accordingly, many companies exclude CNS tumors in clinical trial inclusion criteria for early development of new molecules and will only pursue drugs when brain tumors are considered as a 'drug-line extension'. Additionally, the requisite regulatory path has not been defined for some rare mid- and low-grade gliomas and medulloblastoma. 
The path to commercialization has been historically slow. For TMZ, a relatively straightforward monofunctional alkylating agent, the path started in the 1970s in the Institute of Cancer Research in London. It had formulation problems in the 1980s and was licensed to Schering Plough in 1997. While approved for recurrent/ progressive anaplastic astrocytoma in 1999, it was not approved for GBM until 2005.

There is some concern that big pharma is not likely to be a major player in CNS cancer drug discovery/development because of internal company competition and prioritization. On the other hand, a great deal of innovation in big pharma is a result of partnered efforts with smaller companies that are willing to focus on concentrated resources to develop a drug that the small company strongly believes in, so we anticipate that big pharma will remain critical to CNS anticancer drug development. To expedite new CNS anticancer drug development, we also believe that it is important to clarify and unite around elucidating a clear regulatory path and establishing clinically meaningful end points. Following the examples of successful partnerships such as those between the Multiple Myeloma Foundation and Celgene Corporation or the Cystic Fibrosis Foundation and Vertex Pharmaceuticals may be useful in advancing new strategies to develop brain tumorspecific drugs. Lastly, industry-academic relationship will always be important and necessary for cancer drug development [17].

\section{Drug targets \& brain penetrant drugs}

Talks in this session focused on specific drugs that are under study for the treatment of brain cancers as well as approaches to enable effective brain penetration of small molecules. One drug, a third-generation mTOR complex 1 (mTORC1) is a selective inhibitor that provides dual-site inhibition of mTORC1 (protein-protein binding of rapamycin combined with an ATP site inhibitor) [18]. The inhibitor crossed the $\mathrm{BBB}$ and achieved tumor growth inhibition in a genetically engineered mouse model (GEMM) with an intact BBB. This third-generation mTORC1 inhibitor has improved PD over time in contrast with rapidly reversibly binding mTOR inhibitors, potentially suggesting an improved residence time.

Based on the molecular relationship between the $I D H 1$ gene and its mutation status and survival in gliomas, IDH1 protein inhibitors are under clinical development. These studies tend to focus on low- and mid-grade gliomas and, therefore, may require surrogate end points for trials in lower grade gliomas to shorten the clinical trial development period and pharmaceutical risk since median PFS and median OS may pose considerable cost risks to a company as they lead to long duration studies in low- and mid-grade gliomas compared with GBM studies. In addition, there may be challenges in patient selection and developing effective combination therapies for IDH1 inhibitors. The importance and risk of drug combination studies for low- and midgrade gliomas is real and substantial in today's regulatory environment.

Another drug under preclinical investigation addresses treatment of MYC-driven tumors using inhibitors of glutamine metabolism. These inhibitors attenuate growth of MYC-driven medulloblastoma cell lines in vitro and increased survival in in vivo xenograft models. An example offered was that of prodrugs of 6-diazo5-oxo-norleucine, a glutamine antagonist, that were used to improve oral absorption and brain penetration, and led to improved survival in an orthotopic xenograft brain tumor [19].

L-type amino acid transporter (LAT1) expression is highly upregulated in tumors and increases with tumor grade. LAT1 is expressed on the luminal and abluminal membranes of microvessels comprising the $\mathrm{BBB}$. This observation led investigators to study molecules that utilize LAT1 as an uptake transporter to achieve brain- and tumor-specific concentration of drug QBS10072S (Quadriga Biosciences Inc.). This agent showed tumor cell specific cytotoxicity in vitro that increased with LAT1 expression and led to less toxicity to normal cells than did non-LAT1-directed mustards. In orthotopic GBM mouse models, QBS10072S showed a significant survival benefit, independent of O6- methylguanine-DNA methyltransferase (MGMT) expression, without affecting body weight or white blood cell counts during 4 weeks of dosing. Further study will be needed to determine whether it will go into clinical trials.

Imaging of drugs \& drug effects in the CNS Noninvasive imaging is a powerful approach for determining drug biodistribution, treatment effects and studying tumor progression in humans. Presentations in this session focused on two principal imaging modalities: PET and MRI. Each approach has its strengths and 
limitations. PET requires the synthesis of radiotracers, which often involves incorporating carbon-11 ( $\left.\mathrm{t}_{1 / 2}: 20.4 \mathrm{~min}\right)$ or fluorine-18 $\left(\mathrm{t}_{1 / 2}\right.$ : $110 \mathrm{~min}$ ) into the target molecule. Following injection, the biodistribution of the radiotracers can be quantitatively assessed using PET imaging. This approach is quantitative and highly sensitive, however, spatial accuracy is limited to 1-2 mm. In contrast, MRI has very high spatial accuracy, but is generally less sensitive than PET.

One of the themes in the session was the development and use of imaging methods that focus on the metabolic abnormalities found in tumor cells. Since pyruvate kinase M2 catalyzes the final and rate-limiting step in tumor glycolysis, the PET radiotracer $\left[{ }^{11} \mathrm{C}\right]-\mathrm{DASA}-23$, has been used for noninvasive quantification of GBM-associated pyruvate kinase M2 expression. In addition, other approaches have been developed to detect tumor cells based on mutations in IDH that affect pyruvate metabolism in GBM. To this end, one can use hyperpolarized ${ }^{13} \mathrm{C}$ nuclear magnetic resonance to increase the sensitivity of MRI for detecting metabolites. For instance, hyperpolarized $\left[1-{ }^{13} \mathrm{C}\right]$-pyruvate can be used to monitor changes in the flux of pyruvate into lactate and the tricarboxylic acid cycle in tumor cells compared with normal tissue or in response to therapy. IDH1 mutations result in excess production of the metabolite 2-hydroxyglutarate (2HG), therefore, one can use $2 \mathrm{D}$ correlation nuclear magnetic resonance spectroscopy to follow the change in $2 \mathrm{HG} \mathrm{lev-}$ els in pre- and post-treatment gliomas [20]. Also, ${ }^{13} \mathrm{C}$-magnetic resonance spectroscopy using hyperpolarized $\left[1-{ }^{13} \mathrm{C}\right] \alpha$-ketoglutarate can be used to detect $2 \mathrm{HG}$ production and changes in enzyme activities caused by $I D H 1$ mutants including BCAT1 [2].

MRI is the mainstay for detecting pathophysiologic changes associated with tumors in the CNS. For example, MRI has been used to correlate perfusion and response to anti-angiogenic therapies. There is a strong general agreement that drug penetration of the CNS is a critical issue for the effective treatment of primary and secondary brain tumors. Some aspects of drug penetration can be mathematically modeled and correlated with drug delivery during anti-angiogenic therapy based on simultaneous PET/MRI information. An example of this approach was the measurement of $\left[{ }^{11} \mathrm{C}\right]-\mathrm{TMZ}$ penetration, preand post-bevacizumab therapy, and analysis of blood flow, permeability and diffusion through the tumor microenvironment associated with this treatment [21].

The development of imaging probes that bind to specific tumor-associated proteins is another intense area of research. An example of this is reflected in the development of Martinostat, an ${ }^{11} \mathrm{C}$-labeled hydroxamate PET radiotracer that targets histone deacetylase in tumor cells. An underlying theme of this work is that extensive structure-activity relationship analyses are required to identify a brain-penetrant imaging agent and the translational value of a molecular imaging agent. In addition, there are other tumor-targeted PET agents including $\left[{ }^{18} \mathrm{~F}\right]$-FPPRGD2, a fluorine-18 labeled RGD peptide, and ${ }^{18} \mathrm{~F}-\mathrm{FSPG}$, a glutamate analog. The guanine derivative $\left[{ }^{18} \mathrm{~F}\right]$-FHBG has been used to detect cytolytic $\mathrm{T}$ cells genetically engineered to express the interleukin 13-zetakine gene, which encodes a receptor protein that targets these $\mathrm{T}$ cells to tumor cells, and the herpes simplex virus 1 thymidine kinase suicide gene, which phosphorylates $\left[{ }^{18} \mathrm{~F}\right]$-FHBG but also undergoes programmed cell death when exposed to the drug ganciclovir [22]. In summary, this session presented a cross-section of state-of-the-art imaging methods applied to detect brain tumors, monitor response to therapy and determine drug penetration. It is a developing area that may be critical to the future development of surrogates of drug action and efficacy that will allow more rapid and precise drug and drug combination development and regulatory approval in the future.

\section{New types of drugs \& drug delivery opportunities}

The inability to achieve therapeutic concentrations of pharmaceutical agents in the brain is believed to be the major challenge in developing drugs for CNS tumors. This challenge arises in large part because of the $\mathrm{BBB}$, including tight junctions between endothelial cells, and the expression of efflux pumps that limit the free passage of systemically delivered therapies from the bloodstream to tumors within the CNS. Topics in this session covered new types of drugs and drug delivery approaches that could putatively overcome the major hurdle of drug tissue penetration for brain tumor therapy.

One area of research focuses on nanoparticles and polymer-based devices used to encapsulate, and thereby control, binding and release of active pharmaceutical agents for the treatment of CNS cancers. This research requires an 
advanced understanding of nanoparticle delivery mechanisms and assessing how drug encapsulation affects movement across the BBB [3]. Highlighted in this discussion were options to alter the size and loading of nanoparticles, such as varying the compositions of poly (lactic-coglycolic) acid-based nanoparticles as a means to control drug concentrations in the CNS. One can also take advantage of imaging methods to track nanoparticle distribution in tumors and brain. Also discussed were delivery approaches to allow nanoparticle drug entry into the CNS based on pathogen-inspired mechanisms [23].

Another approach, a dual moiety drug delivery system, was discussed wherein Toca 511, a replicating retroviral vector that encodes cytosine deaminase, and Toca FC, an extended release 5-fluorocytosine that serves as a prodrug activated by cytosine deaminase produce 5-fluoruracil, a cytotoxic molecule. Toca 511 was given to patients intravenously prior to surgical tumor removal. Using this approach, successful gene transduction was observed in $82 \%(14 / 17)$ patients in a clinical study. Independent radiology review showed $9 \%(1 / 11)$ had a radiologic complete response and $18 \%(2 / 11)$ had stable disease. In addition, this approach was shown to be effective in flank and intracerebral gliomas in mice, accompanied by an upregulation of $\mathrm{CD} 4$ and CD8 T cells and reduction of immunosuppressive myeloid cells. This finding prompted studies that examined combinations of the Toca 511/FC system with immune checkpoint inhibitors, $\alpha$ PD- 1 and $\alpha$ CTLA- 4 , also in syngeneic mouse models, to determine how best to combine these treatments. Early results appeared promising based on efficacy and low toxicity [24] and may lead to future clinical trials.

Another approach that temporarily disrupts the intact BBB involves using laser interstitial thermal therapy. Experiments have shown that BBB disruption may persist for 4-6 weeks following a single hyperthermia treatment, as measured by MRI and separately by quantifying serum levels of brain-specific enolase. Early data on the first 14 patients studied were presented suggesting that laser interstitial thermal therapy can cause a marked disruption in the $\mathrm{BBB}$, which may improve the efficacy of a chemotherapy agent having historically poor CNS penetration, such as doxorubicin [25].

Additional presentations included discussion of new compounds or targets that may be used for CNS anticancer drug development. An experimental compound, NEO214, was created by conjugating an anti-inflammatory PDE 4 inhibitor, rolipram to perillyl alcohol. NEO214, unlike unconjugated rolipram, demonstrates relatively good tolerability in animals (up to $200 \mathrm{mg} / \mathrm{kg}$ ). NEO214 was also shown to prolong survival in an intracranial tumor model, with U251 TMZ-resistant glioma cells implanted into the frontal cortex of athymic nude mice. Along with HPLC data, the mouse studies suggested that NEO214 can cross the BBB. The results demonstrated that NEO214 is an effective drug against a wide range of different TMZ-resistant gliomas in preclinical models.

Another new drug approach was based on exploiting monoamine oxidase $\mathrm{B}(\mathrm{MAOB})$, an enzyme that is significantly upregulated in astrocytoma and glioma. To better understand the value of MAOB, two strategies were proposed: the use of $\mathrm{MAOB}$ to: convert prodrugs into conventional agents, like the MGMT inhibitor $\mathrm{O}^{6}$ benzylguanine; and into active agents that target mitochondrial DNA (so-called 'mitochondrial smart bombs'). Both strategies were shown to increase survival in mouse intracranial GBM models [26].

While there is a great deal of general interest in science and commerce for the CRISPR (clustered regularly interspaced short palindromic repeats) technology, not much has been seen that is directly applicable to CNS cancers. It was, therefore, of some interest that one presentation dealt with the use of CRISPR-Cas9 and RNAi technologies to identify novel molecular targets for GBM. From this research using patient tumor isolates, different novel targets were identified including a potentially druggable kinase, PKMYT1. Further study using specific agents will be required to determine the value of this target for CNS cancer treatment.

Another approach, presented as preliminary preclinical data, looked at combining ibudilast (MN-166) with TMZ for GBM. This research found that high levels of the macrophage inhibitory factor (MIF) and its receptor CD74 were associated with poor OS in GBM patients. Furthermore, when ibudilast was used as an inhibitor of MIF expression, it was found that ibudilast combined with TMZ resulted in significant blockage of MIF expression, increased apoptosis and longer survival in patient-derived xenograft models. Based on these data, a Phase I/II clinical trial for recurrent GBM is being proposed. 
Modeling drug effects \& tumor growth CNS cancer is experiencing an explosion of technological advances in genomics, proteomics and epigenetics that are providing a foundation for molecular classifications, and new drug targets and associated therapies. These advances have given rise to global initiatives in personalized and precision medicine that are likely to benefit from, and even require, insights from mathematical models, and animal models that often provide the data used in mathematical models. The functional phenotypic realization of a given mutation depends intimately on its context, therefore, a key emerging issue for precision medicine is how to connect genomic data with phenotypic outcomes. Until recently, integration of mathematical models with cancer therapy, particularly in the clinical domain, has mostly been in the realm of PK and PD modeling. In that arena, patient plasma drug concentrations and biomarkers could be used as guides to rational therapy, as well as accounting for interpatient variability. As genetic and, to a more limited extent, proteomic analyses become standard and form the basis for stratification of subpopulations of patients, there will be new opportunities to strategize on how mathematical models apply to patient therapy. The modeling session captured some of these novel modeling approaches.

Every mathematical modeling approach invariably relies on preclinical tumor models and/or patient data. There are an increasing number of choices of preclinical mouse models available for preclinical studies of brain tumors. These include orthotopic patient-derived xenografts (i.e., PDX models), GEMMs, and nongermline GEMMs (nGEMMs), in which tumors are generated by introduction of oncogenes or oncogene-expressing cells into the brain $[27,28]$. Each of these has been applied to medulloblastoma to evaluate therapies. Both the GEMMs and nGEMMs can incorporate one or two genetic modifications, whereas PDX models can display the range of genetic changes seen in the original patient tumors. nGEMMs are advantageous in that they can be created relatively quickly (i.e., without generating an entirely new line of mice). Regardless of the tumor model, studies show differences in drug efficacy between flank versus intracerebral sites. This may be due to limitations of drug penetration across the $\mathrm{BBB}$, as well as effects of the tumor microenvironment on drug trafficking and metabolism. Identification of the causes of drug failures in brain tumor models is a critical step in recognizing approaches to enhancing drug penetration into brain tumors. Strategies ranging from chemical modification of drugs to nanoparticle encapsulation, as mentioned earlier, may ultimately allow therapeutic agents to cross the BBB and persist long enough to exert their effects on tumor cells.

Other speakers at the conference focused on current state-of-the-art mathematical modeling approaches being developed to improve drug therapies and early clinical trial designs. This included phenotypic cell models driven by MRI imaging scale metrics. This approach has led to a proliferation-invasion (PI) model that utilizes patient MRI data to uniquely estimate glioma cell density using proliferation and diffusion (invasion) rate parameters [29,30]. The PI model has been extended to a proliferation-invasion-hypoxia-necrosisangiogenesis model specifically used to analyze anti-angiogenic therapy and the need for gross total resection. Again, the phenotypic nature of the model is due to tracking of four cell populations; normoxic, hypoxic, necrotic and vascular or endothelial cells. The models appear flexible in their ability to be used in silico as well as with patient measurements. The models are meant to be patient-specific and due to the resolving capability of MRI can be used to analyze regional changes in each cell population. Another phenotypic cell modeling approach is an autophagy-resistance model that assessed three cell populations; nonautophagy, physiological autophagy and quiescent autophagy, and their interconnected dynamics to determine improved dosing schedules to avoid drug resistance to kinase inhibitors for melanoma patients [31]. This model was the foundation for a new modeling endeavor called Phase I Imaginary that can distinguish among diverse virtual patient cohorts and their varied responses under different treatment conditions and ultimately lead to new treatment and dosing recommendations. This investigation represents an important trend in translational medicine through bridging of preclinical and clinical drug development paths via mathematical models to generate virtual patient populations that can be stratified appropriately before drugs are ever given to patients.

It was also shown that systems pharmacological and mechanistic mathematical models can be applied to evaluate drug therapies in virtual GBM patient populations. These can be viewed as large-scale PK/PD models, which rely on chemical and enzyme kinetics to characterize cell signaling cascades and drug interventions. A GBM systems based model that was discussed 
considers major pathways integral to GBM, such as receptor tyrosine kinases, proliferation, p53 DNA damage response and cell-cycle data using large public databases such as The Cancer Genome Atlas, as well as patient cell based measurements. The GBM model has 781 protein species and 1738 reactions that will undergo various validations in cell systems and unit testing of each submodel. Since patients in the Cancer Genome Atlas have unique genomic profiles that are incorporated into the model, the ability to perform virtual patient simulations is quite doable. To evaluate drug therapies, the appropriate PK models can be linked to a system-based PD model with resultant population simulations providing key insights into drug successes and failures, which potentially could identify subpopulations that would most likely benefit from specific drug therapies.

The net message from the session is that new tools and systems are being developed that will advance the application of quantitative approaches to improve therapies for GBM patients. The mathematical modeling approaches that highlighted the session contrasted two evolving strategies, phenotype-based and mechanics-based, to impact clinical decision making for individual patients. Each approach has its limitations in terms of inherent assumptions, data collection and model uncertainty, yet at the same time these approaches are needed to achieve the true potential of precision medicine.

\section{Drug targets \& CNS tumor \\ microenvironment}

Glioblastoma was formerly called glioblastoma multiforme. Multiforme in that context, referred to the relatively undifferentiated and anaplastic nature of the tumor cells, as well as marked pleomorphism, necrosis and proliferative endothelial cells. Although the WHO simplified the naming of this tumor in its 2007 edition, the word 'multiforme' captures the importance of heterogeneity in this tumor type. Immune and stromal cells comprise a third or more of the cells in GBM tumors. Work by many laboratories is focused on understanding cross-talk among cells in tumors and cells in the tumor microenvironment, how these interactions drive progression and whether any part of this cross-talk is potentially targetable.

Microglia are one component of the glioma tumor microenvironment. Based on studies of the GBM tumor microenvironment, it can be shown that GBM cells express cell surface molecules that suppress the immunologic function of microglia, while microglia reciprocally secrete specific cytokines to stimulate GBM growth [32]. These interactions are mediated in-part through secreted microvesicles, while PI3K $\gamma$ regulates recruitment and protumor properties in microglia, suggesting a targetable strategy through inhibition of PI3K $\gamma$. With the appropriate $\operatorname{drug}(s)$, this approach may lead to a clinical trial.

An interesting study using a rodent breast cancer model demonstrated reduced expression of phosphate and tensin homolog in cancer cells from breast cancer cells of brain metastasis compared with extracranial breast tumor cells. Furthermore, it was shown that astrocytederived exosomal miR-19a reversibly downregulated Phosphate and tensin homolog expression in breast-brain metastases, leading to increased secretion of CCL2, recruitment of myeloid cells and promotion of metastases. Ablation of CCL2 inhibited brain metastasis in vivo, suggesting the potential for using CCL2 inhibitors as a therapeutic approach [33].

In novel experiments, OMX, a pegylated hemoprotein trimer derived from thermophilic bacteria, is used as an oxygen delivery vehicle to oxygenate rodent tumors and thereby serving as a therapeutic strategy for the hypoxic tumor microenvironment in GBM. To this end, using an orthotopic GL261 rodent tumor model, OMX (an IND stage drug candidate) reversed the immunosuppressive microenvironment leading to increased T-cell infiltration, increased interferon $\gamma$ inducible cytokines and improved survival in response to anti-PD1 therapy.

In addition to new drugs for the treatment of CNS tumors, repurposing drugs for gliomas was also discussed. One such drug is dianhydrogalactitol (VAL-083; DelMar Pharmaceuticals) a repurposed alkylating agent that accumulates in brain tumor tissue, and promotes interstrand crosslinks at guanine- $\mathrm{N}^{7}$, leading to cell death. VAL-083 is approved in China for treatment of CML and lung cancer. VAL-083 showed efficacy in MGMT nonmethylated GBM cell lines with broad clinical activity, and was well tolerated in a Phase I/II clinical trial in patients with recurrent GBM. Several Phase II trials and a Phase III clinical trial are planned or underway.

FLG-003 (FLAG Therapeutics, Inc.) is another repurposed drug. A water soluble anti-angiogenic and antitubulin agent, it had 
been discarded as a breast cancer candidate due to CNS side effects. Repurposing of this agent as a GBM therapeutic demonstrated efficacy in multiple patient cell-derived orthotopic PDX models. A clinical trial is planned for early 2017.

While immunotherapy for CNS tumors was not addressed in the conference, one talk addressed PDL1 expression in meningioma. Binding of PDL1 with the monoclonal antibody avelumab led to antibody-dependent cellmediated cytotoxicity by binding CD16 on natural killer (NK) T cells. High avidity NK cells engineered to express high avidity CD16 and high level expression of IL-2, killed meningioma via antibody-dependent cell-mediated cytotoxicity with avelumab. Both hypoxia and interferon $\gamma$ led to increased expression of PDL1 and enhanced high avidity NK killing of cultured meningioma cells. Validation in animal experiments is underway and, if successful, might lead to a future clinical trial.

This session highlighted how interactions between tumor cells and cells in the microenvironment contribute to malignancy in brain cancers, and offered a few new therapeutics that target tumor cells or that block tumorpromoting interactions among tumor cells and immune and endothelial cells in the tumor microenvironment.

\section{Conclusion}

The second CADDDC addressed new approaches to understanding how high-grade gliomas, (GBM and medulloblastoma), grow and which new target and drug modeling approaches might provide future purchase for new drug development. Balancing this growing optimism, the conference also addressed regulatory and financial risks that complicate the development of new drugs to treat CNS tumors. Some speakers and discussants believe that the long trajectory and multiple challenges to novel drug discovery, and expectation that more than one drug will be needed to adequately inhibit critical intracellular tumor pathways, will lead to a plethora of financial risk without changes to patent and/or orphan drug exclusivity for most pharmaceutical/biotechnology companies, posing major disincentives to progress. That was believed to be the case for the rare gliomas other than GBM and medulloblastoma tumors. Others expressed the position that companies would come forward if better target identification was coupled with better drugs and, especially, if the drug target extended to other cancers and, therefore, a larger market.

While there were few consensuses from the conference, one that did emerge is the need for continued and focused discussion among academic and industry scientists and clinicians to address tumor targets, new drug chemistry and more time- and cost-efficient clinical trials' methodologies based on surrogate end points. Because of these challenges and the enthusiasm of attendees, Society for Neuro-Oncology will plan a third CADDDC in November 2018.

\section{Acknowledgements}

The authors wish to thank Joann Aaron for her careful and thoughtful editing.

\section{Diclaimer}

Funding for the second CADDDC meeting was provided by Genentech, Bristol-Myers Squibb, AbbVie Inc., Pershing Square Sohn Cancer Research Alliance and Agios Pharmaceuticals.

Financial \& competing interests disclosure

The authors have no relevant affiliations or financial involvement with any organization or entity with a financial interest in or financial conflict with the subject matter or materials discussed in the manuscript. This includes employment, consultancies, honoraria, stock ownership or options, expert testimony, grants or patents received or pending, or royalties.

No writing assistance was utilized in the production of this manuscript.

\section{References}

1 Levin VA, Tonge PJ, Gallo JM et al. CNS anticancer drug discovery and development conference white paper. Neuro. Oncol. 17(suppl. 6), vi1-vi26 (2015).

2 Chaumeil MM, Larson PE, Woods SM et al. Hyperpolarized [1-13C] glutamate: a metabolic imaging biomarker of IDH1 mutational status in glioma. Cancer Res. 74(16), 4247-4257 (2014).
3 Cook RL, Householder KT, Chung EP, Prakapenka AV, DiPerna DM, Sirianni RW. A critical evaluation of drug delivery from ligand modified nanoparticles: Confounding small molecule distribution and efficacy in the central nervous system. J. Control. Release 220 (Pt A), 89-97 (2015).

4 Ostrom QT, Gittleman H, Fulop J et al. CBTRUS statistical report: primary brain and central nervous system tumors diagnosed in the United States in
2008-2012. Neuro. Oncol. 17(Suppl. 4), iv1-iv62 (2015).

5 Nayak L, Lee EQ, Wen PY. Epidemiology of brain metastases. Curr. Oncol. Rep. 14(1), 48-54 (2012).

6 The Cancer Genome Atlas Research Network. Comprehensive genomic characterization defines human glioblastoma genes and core pathways. Nature 455 , 1061-1068 (2008). 


\section{CNS drug discovery/development CONFERENCE REPORT}

7 Taylor MD, Northcott PA, Korshunov A et al. Molecular subgroups of medulloblastoma: the current consensus. Acta Neuropathol. 123(4), 465-472 (2012).

8 Westerhout J, van den Berg DJ, Hartman R, Danhof M, de Lange EC. Prediction of methotrexate CNS distribution in different species - influence of disease conditions. Eur. J. Pharm. Sci. 57 11-24 (2014).

9 Yamamoto Y, Valitalo PA, van den Berg DJ et al. A generic multi-compartmental CNS distribution model structure for 9 drugs allows prediction of human brain target site concentrations. Pharm. Res. 34(4), 333-351 (2016).

10 Lu H, Tonge PJ. Drug-target residence time: critical information for lead optimization. Curr. Opin. Chem. Biol. 14(4), 467-474 (2010).

11 Walkup GK, You Z, Ross PL et al. Translating slow-binding inhibition kinetics into cellular and in vivo effects. Nat. Chem. Biol. 11(6), 416-423 (2015).

12 Wu P, Nielsen TE, Clausen MH. FDAapproved small-molecule kinase inhibitors. Trends Pharmacol. Sci. 36(7), 422-439 (2015).

$13 \mathrm{Ou} \mathrm{SH}$, Ahn JS, De Petris L et al. Alectinib in crizotinib-refractory ALK-rearranged non-small-cell lung cancer: a Phase II global study. J. Clin. Oncol. 34(7), 661-668 (2016).

14 Camidge DR, Bazhenova L, Salgia P, Al E. Safety and efficacy of brigatinib (AP26113) in advanced malignancies, including ALK1 non-small cell lung cancer (NSCLC). J. Clin. Oncol. 33(Suppl.), Abstr 8062 (2015).

15 Budish E, Roin BN, Williams H. Do firms underinvest in long-term research? Evidence from cancer clinical trials. Am. Econ. Rev. 105(7), 2044-2085 (2015).

16 Gaddipati H, Liu K, Pariser A, Pazdur R. Rare cancer trial design: lessons from FDA approvals. Clin. Cancer Res. 18(19), 5172-5178 (2012).
17 Vallance P. Industry-academic relationship in a new era of drug discovery. J. Clin. Oncol. 34(29), 3570-3575 (2016).

18 Rodrik-Outmezguine VS, Okaniwa M, Yao Z et al. Overcoming mTOR resistance mutations with a new-generation mTOR inhibitor. Nature 534(7606), 272-276 (2016).

19 Hanaford AR, Archer TC, Price A et al. Discovering innovative therapies for rare tumors: combining genetically accurate disease models with in silico analysis to identify novel therapeutic targets. Clin. Cancer Res. 22(15), 3903-3914 (2016).

20 Jafari-Khouzani K, Loebel F, Bogner W et al. Volumetric relationship between 2-hydroxyglutarate and FLAIR hyperintensity has potential implications for radiotherapy planning of mutant IDH glioma patients. Neuro Oncol. 18(11), 1569-1578 (2016).

21 Batchelor TT, Gerstner ER, Emblem KE et al. Improved tumor oxygenation and survival in glioblastoma patients who show increased blood perfusion after cediranib and chemoradiation. Proc. Natl Acad. Sci. USA 110(47), 19059-19064 (2013).

22 Yaghoubi SS, Jensen MC, Satyamurthy N et al. Noninvasive detection of therapeutic cytolytic T cells with 18F-FHBG PET in a patient with glioma. Nat. Clin. Prac. 6(1), 53-58 (2009).

23 Mccall RL, Cacaccio J, Wrabel E, Schwartz ME, Coleman TP, Sirianni RW. Pathogeninspired drug delivery to the central nervous system. Tissue Barriers 2(4), e944449 (2014).

24 Yagiz K, Huang TT, Lopez Espinoza F et al. Toca 511 plus 5-fluorocytosine in combination with lomustine shows chemotoxic and immunotherapeutic activity with no additive toxicity in rodent glioblastoma models. Neuro. Oncol. 18(10), 1390-1401 (2016).

25 Leuthardt EC, Duan C, Kim MJ et al. Hyperthermic laser ablation of recurrent glioblastoma leads to temporary disruption of the peritumoral blood brain barrier. PLoS ONE 11(2), e0148613 (2016).

26 Sharpe MA, Livingston AD, Gist TL, Ghosh P, Han J, Baskin DS. Successful treatment of intracranial glioblastoma xenografts with a monoamine oxidase B-activated pro-drug. EBioMedicine 2(9), 1122-1132 (2015).

27 Pei Y, Liu KW, Wang J et al. HDAC and $\mathrm{PI} 3 \mathrm{~K}$ antagonists cooperate to inhibit growth of MYC-driven medulloblastoma. Cancer Cell 29(3), 311-323 (2016).

28 Markant SL, Esparza LA, Sun J et al. Targeting sonic hedgehog-associated medulloblastoma through inhibition of Aurora and Polo-like kinases. Cancer Res. 73(20), 6310-6322 (2013).

29 Baldock AL, Ahn S, Rockne R et al. Patient-specific metrics of invasiveness reveal significant prognostic benefit of resection in a predictable subset of gliomas. PLoS ONE 9(10), e99057 (2014).

30 Jackson PR, Juliano J, Hawkins-Daarud A, Rockne RC, Swanson KR. Patient-specific mathematical neuro-oncology: using a simple proliferation and invasion tumor model to inform clinical practice. Bull. Math. Biol. 77(5), 846-856 (2015).

31 Kim E, Rebecca VW, Smalley KS, Anderson AR. Phase I trials in melanoma: a framework to translate preclinical findings to the clinic. Eur. J. Cancer 67 213-222 (2016).

32 Kozono D, Li J, Nitta M et al. Dynamic epigenetic regulation of glioblastoma tumorigenicity through LSD1 modulation of MYC expression. Proc. Natl Acad. Sci. USA 112(30), E4055-4064 (2015).

33 Chang AL, Miska J, Wainwright DA et al. CCL2 produced by the glioma microenvironment is essential for the recruitment of regulatory $\mathrm{T}$ cells and myeloid-derived suppressor cells. Cancer Res 76(19), 5671-5682 (2016).

34 US Food \& Drug Administration. Novel drug approvals for 2015. www.fda.gov 